\title{
FOCUS: an Alignment-free Model to Identify Organisms in Metagenomes Using Non-negative Least Squares
}

One of the major goals in metagenomics is to identify the organisms present in a microbial community from unannotated shotgun sequencing reads. Taxonomic profiling has valuable applications in biological and medical research, including disease diagnostics. Most currently available approaches do not scale well with increasing data volumes, which is important because both the number and lengths of the reads provided by sequencing platforms keep increasing. Here we introduce FOCUS, an agile composition based approach using nonnegative least squares (NNLS) to report the focal organisms present in metagenomic samples and profile their abundances. FOCUS was tested with simulated and real metagenomes, and the results show that our approach accurately predicts the organisms present in microbial communities. FOCUS was implemented in Python. The source code and web-sever are freely available at http://edwards.sdsu.edu/FOCUS. 
1

2 FOCUS: an Alignment-free Model to Identify Organisms in Metagenomes Using Non-

3 negative Least Squares

4

5 Genivaldo Gueiros Z. Silva ${ }^{1}$, Daniel A. Cuevas $^{1}$, Bas E. Dutilh ${ }^{4,5}$, and Robert A. Edwards ${ }^{1,2,3,5,6}$ 6

$7{ }^{1}$ Computational Science Research Center, ${ }^{2}$ Department of Computer Science, and ${ }^{3}$ Department of 8 Biology, San Diego State University, 5500 Campanile Drive, San Diego, CA 92182, USA, $9{ }^{4}$ Centre for Molecular and Biomolecular Informatics, Radboud Institute for Molecular Life 10 Sciences, Radboud University Medical Centre, Geert Grooteplein 28, 6525 GA, Nijmegen, The 11 Netherlands, ${ }^{5}$ Department of Marine Biology, Institute of Biology, Federal University of Rio de 12 Janeiro, Brazil, ' Division of Mathematics and Computer Science, Argonne National Laboratory, 139700 S. Cass Ave, Argonne, IL 60439, USA

14

$15 *$ For correspondence please contact Dr. Robert Edwards at redwards@mail.sdsu.edu. 


\section{Abstract}

18 One of the major goals in metagenomics is to identify the organisms present in a microbial 19 community from unannotated shotgun sequencing reads. Taxonomic profiling has valuable

20 applications in biological and medical research, including disease diagnostics. Most currently 21 available approaches do not scale well with increasing data volumes, which is important because

22 both the number and lengths of the reads provided by sequencing platforms keep increasing. Here we 23 introduce FOCUS, an agile composition-based approach using non-negative least squares (NNLS) to 24 report the focal organisms present in metagenomic samples and profile their abundances. FOCUS 25 was tested with simulated and real metagenomes, and the results show that our approach accurately 26 predicts the organisms present in microbial communities. FOCUS was implemented in Python. The 27 source code and web-sever are freely available at http://edwards.sdsu.edu/FOCUS.

Introduction

30 Microbes are more abundant than any other cellular organism (Whitman et al. 1998), and

31 it is important to understand which organisms are present and what they are doing (Handelsman 32 2004). In many environments a majority of the microbial community members cannot be

33 cultured, and metagenomics is a powerful tool to directly probe uncultured genomes and 34 understand the diversity of microbial communities by using only their DNA (Sharon and Banfield 35 2013).

36 Understanding microbial communities is important in many areas of biology. For 37 example, metagenomes can distinguish taxonomic and functional signatures of microbes 38 associated with marine animals (Trindade-Silva et al. 2012) or disease states (Belda-Ferre et al. 39 2012). Large sequencing volumes, short read lengths, and sequencing errors make the task of 
40 identifying the diversity of organisms present in metagenomes challenging (Mande et al. 2012).

41 Many programs exist for this, and they are either homology- or composition-based.

42 Homology-based programs normally use the BLAST program (Altschul et al. 1997) to

43 identify the best hit in a large database output. In MG-RAST (Meyer et al. 2008) sequences are 44 aligned to a set of databases in order to classify the metagenomic sample. MetaPhlAn (Segata et 45 al. 2012) and GenomePeek (McNair and Edwards) use a reduced database containing only 46 marker genes, e.g., unique clades and housekeeping genes, allowing the BLAST search to be fast. 47 PhymmBL (Brady and Salzberg 2011) improves the BLAST results using interpolated Markov 48 models. GASiC (Lindner and Renard 2013) uses Bowtie (Langmead et al. 2009) and the 49 reference genomes similarities to correct the observed abundance estimated. Parallel-Meta (Su et al. 2012) a fast program, which requires a GPU, uses megaBLAST (Zhang et al. 2000) and HMM 51 (Hidden Markov Model) to improve the homology result. Most of these applications classify 52 sequences individually, and generate a taxonomic profile by summing the bins.

In general, composition-based approaches use oligonucleotide ( $k$-mer) frequencies. Taxy

54 (Meinicke et al. 2011) uses oligonucleotide distribution in metagenomes and in reference

55 genomes and uses mixture modeling to identify the organisms present in the metagenome, and 56 RAIphy (Nalbantoglu et al. 2011) identifies organisms using oligonucleotides and relative 57 abundance index.

58 We developed a new approach that reconstructs a taxonomic profile using an ensemble $k$ 59 mer composition of the entire metagenome. We compute the optimal set of organism abundances 60 using non-negative least squares (NNLS) to match the metagenome $k$-mer composition to 61 organisms in a reference database. $K$-mers have previously been used to cluster unknown 62 sequences (Teeling et al. 2004; McHardy et al. 2007) and NNLS has been used to identify the 63 genera present in metagenomic samples based on variations in gene count (Carr et al. 2013). Here 64 we combine these two approaches in FOCUS, an ultra fast, accurate, composition based approach 
65 to identify the taxa present in a metagenome. We compare the performance of FOCUS to GASiC,

66 MetaPhlAn, RAIphy, PhymmBL, Taxy, and MG-RAST.

68 Methods

69 FOCUS workflow is described in Figure 1. As in most composition-based approaches, a

70 training set is pre-generated using the complete genomes information, and here the non-negative

71 least squares (NNLS) is applied to compute the relative abundance of each organism in the 72 database into the unknown data.

\section{Reference dataset}

FOCUS requires a group of reference genomes to model and identify the organisms present in a metagenome. 2,766 complete genomes were downloaded from the SEED servers (Aziz et al. 2012) on 20 December 2013 (see Supplementary Table 1). $K$-mer frequencies (k=6-8, default: $\mathrm{k}=7$ ) were calculated for both strands using Jellyfish 1.1.6 (Marçais and Kingsford 2011),

79 reducing the number of dimensions (Strous et al. 2012), and $k$-mer counts were normalized by the sum of frequencies. The user can also create their own training set, which is scalable to the

81 quickly increasing number of available reference genomes because it also uses Jellyfish in the $k$ 82 mer counting.

\section{Simulated and real metagenomes}

85 In order to evaluate FOCUS performance, a simulated dataset of short sequences 86 (SimShort), containing 500,000 single $100 \mathrm{nt}$ reads, was created using the supplied error model 87 for Illumina GA IIx with TrueSeq SBS Kit v5-GA using GemSim (McElroy et al. 2012) 88 (Supplementary Table 2). The previous published high complexity simulated dataset (SimHC) 89 from FAMeS (Mavromatis et al. 2007) was also used in the evaluation. Moreover, real 
metagenomic datasets were selected as test cases: one under healthy conditions, one under

91 disease conditions (MG-RAST accession 4447943.3 and 4447192.3) (Belda-Ferre et al. 2012),

92 one fecal sample from a healthy individual (MG-RAST accession 4440945.3) (Kurokawa et al.

93 2007), and three hundred datasets from the Human Micriobiome Project (HMP) (Consortium

94 2012) (Supplementary Table 3) were selected as a test case.

95

96 Non-Negative Least Squares (NNLS)

The estimation of a parameterized model to understand some data is a fundamental problem in data modeling. Nevertheless, the estimation is not always easy, e.g., in problems like metagenome profiling that cannot have negative values for the fitted parameters. In such case, a solution can be estimated using NNLS, which is defined as:

101 Given a matrix $A \in \mathbb{R}^{\mathrm{mxn}}$ and a vector $\mathrm{b} \in \mathbb{R}^{\mathrm{m}}$, where $\mathrm{m} \geq \mathrm{n}$, find a non-negative vector $\mathrm{x} \epsilon$ $102 \mathbb{R}^{\mathrm{n}}$ to minimize the function (1).

$$
f(x)=1 / 2\|A x-b\|^{2} \text {, where } x \geq 0 \text { and } \sum_{i=i}^{n} x_{i}=1
$$

In FOCUS, the reference matrix $\mathrm{A}$ is composed of $\mathrm{m} k$-mer frequencies from $\mathrm{n}$ genomes,

while a vector describing the user's metagenomic dataset is calculated from the $k$-mer frequencies of both strands from the dataset using Jellyfish. FOCUS uses non-negative least squares to compute the set of $k$-mer frequencies $\mathrm{x}$ that explains the optimal possible abundance of $k$-mers in

110 the user's metagenome by selecting the optimal number of frequencies from the matrix A. We

111 minimize the sum of squared differences (1) using the open source Scipy library (Jones et al.

112 2001) which has a module for the NNLS algorithm which solves the KKT (Karush-Kuhn-Tucker)

113 conditions (Lawson and Hanson 1987). We added Tikhonov regularization (Garda and Galias 114 2012) to deal with genomes that have similar $k$-mer compositions. 


\section{Jackknife resampling of the data}

117 We implemented a jackknife resampling strategy to assess the robustness of the results.

$11850 \%$ of the reads were randomly resampled $1000 \mathrm{x}$, and the species frequencies recalculated. For

119 each species, these 1000 frequencies were averaged and the standard deviation calculated to 120 estimate the spread.

\section{Web-based and graphical user interface version}

As an alternative to the command line version of the program, we have created a user-

123 friendly web version and a graphical user interface (GUI) for Microsoft Windows users. The web 124 server and the GUI are available at http://edwards.sdsu.edu/FOCUS.

\section{Results and Discussion}

\section{Evaluation and comparison with other tools}

128 All tools were run using default parameters and their default reference database, either

129 online (MG-RAST) or using one core on a server with 24 processors x 6 cores Intel(R) Xeon(R)

130 CPU X5650@2.67GHz and 189 GB RAM. We only compared GASiC to the SimHC dataset

131 which had the results previously published (Lindner and Renard 2013). We tried to run the tool;

132 however, it requires a large amount of storage during the process to save its output data.

133 For the real data, three hundred and three metagenomic datasets were selected. First, the

134 metagenomic sample of the human oral cavity from diseased conditions was used. MetaPhlAn 135 apparently over predicted the genera Veillonella due to the short genome, and Taxy did not 136 predict Prevotella hits (see Figure 2) as described in (Belda-Ferre et al. 2012). FOCUS was able 137 to profile the organisms in only 41 seconds. Taxy took about 45 seconds, MetaPhlAn took about 1383 minutes, RAIphy took 52 minutes, MG-RAST took 3 days, and PhymmBL took 1 week and 6 
139 days. Using random subsets for the oral metagenome, we tested the tools scalability and showed

140 that FOCUS and Taxy profile metagenomes in constant time (see Figure 3).

141 The oral metagenome from the healthy condition was used. MetaPhlAn possibly over

142 predicted the genera Neisseria, and Taxy was not able to predict Rothia hits (see Figure 4).

143 FOCUS profiled the metagenome in only 35 seconds. Taxy took about 41 seconds, MetaPhlAn

144 took about 2 minutes, RAIphy took 48 minutes, MG-RAST took 3 days, and PhymmBL took 9 145 days.

146 A fecal metagenome from a healthy individual was used. All the tools predicted that

147 Bifidobacterium and Enterococcus were the two most abundant genera in the sample. However,

148 RAIphy apparently under predicted the genera Bifidobacterium (see Figure 5). For this small

149 dataset, FOCUS profiled the metagenome in 35 seconds. Taxy took about 40 seconds, MetaPhlAn

150 took only 30 minutes, RAIphy took about 4 minutes, MG-RAST took 3 days, and PhymmBL

151 took 2 days and 14 hours.

152 Three hundred metagenomic samples (254 GB total) from HMP were analyzed at all the 153 taxonomy levels using FOCUS (Supplementary Table 4) in about 1 hour and 20 minutes and

154 compared with the published results from MetaPhlAn's paper (Segata et al. 2012) by calculating 155 the Euclidean distance between the results (see Figure 6). For most of the samples, FOCUS and 156 MetaPhlAn have similar predictions at the genera level but vary at the species level. However, for 157 some samples in the posterior fornix and most of the samples from the anterior nares there were 158 differences at all levels which may reflect the additional genome sequencing of isolates from 159 those passages that has occurred since 2012. Other tools were not included in the analysis due to 160 the CPU processing time.

161 For the simulated data, we removed species from the reference dataset that are present in 162 this dataset and tried to predict the genera present in the SimShort dataset. A major limitation of 163 many of the approaches discussed here is that the underlying databases cannot be changed. Only 
164 FOCUS, RAIphy, GASiC, and PhymmBL allow the end user to change their reference database.

165 FOCUS and PhymmBL best predicted the correct genera while RAIphy could not correctly

166 predict their abundance (Figure 7). FOCUS had the fastest performance (45 seconds); RAIphy

167 took about 2 hours, while PhymmBL took approximately 5 days. Supplementary Figure 1 to 5

168 show the same comparison for other taxonomy resolutions.

169 For the SimHC simulated metagenomes, the genera present in the dataset were deleted

170 from the training dataset, and we evaluated the class-level prediction. The tested tools correctly

171 predicted the classes, except that RAIphy over predicted the top two classes (see Figure 8).

172 Again, FOCUS was the fastest tool (30 seconds) in comparison to RAIphy, which took about 1

173 hour and 50 minutes, and PhymmBL, which took about 4 days. See Supplementary Figure 6 to 8

174 for other taxonomy levels.

175 Furthermore, for the SimHC dataset, we ran all the previously used tools and the GASiC 176 published results to evaluate the genera-level prediction. GASiC and PhymmBL had the best 177 predictions, and FOCUS failed in the prediction of 4 minor genera probably because many 178 organisms present in the SimHC dataset were not included in the FOCUS database (see Figure 9).

179 We did not compare the running time because we extracted the GASiC results from its paper;

180 however, in the original paper it took 2 days and needed at least 500 GB of storage to analyze the 181 SimHC simulated metagenome.

182 The very small standard deviations observed after jackknife re-sampling indicate the 183 robustness of our results. Furthermore, in order to show a quantitative evaluation between the real 184 and predicted abundance for the synthetic metagenomes, we computed the Euclidean distance 185 between the real and predicted abundances for all the simulated data presented above (see Figure 186 10). For some of the tools, only genus level predictions are available, but for RAIphy, PhymmBL, 187 and FOCUS we included all taxonomic levels. The data demonstrate that FOCUS had the best 188 prediction in more than half of test cases. 
190 computer. For example, we profiled the real dataset in 1 minute and 45 seconds using an Intel(R)

191 Core(TM) i3@2.53 GHz and 1GB RAM. In addition to the Web server, we have developed a

192 stand-alone version that runs on the Windows ${ }^{\circledR}$ platform.

193

194 Limitations

195 As with other methods created to profile metagenome sequences, FOCUS depends on a 196 curated database of microbial reference genomes in order to predict a specific genus. If a 197 reference genome is absent, the tool will predict the closest reference available.

\section{Conclusions}

200 Here we present FOCUS, an agile solution to identify the organisms present in 201 metagenomic samples that does not rely on mapping individual reads, but instead determines the 202 taxonomic composition of the entire metagenome at once by using NNLS. This makes FOCUS 203 an extremely fast and scalable solution to profile the focal taxa in a metagenome. FOCUS reports

204 very similar species compositions as currently available, state of the art metagenome profiling 205 tools.

\section{Availability and requirements}

208 Project name: FOCUS

209 Project and web server home page: http://edwards.sdsu.edu/FOCUS

210 Operating system: the program has a command line version that works on OS X and Unix, and a 211 GUI for Microsoft Windows users. 
212 Programming language: Python 2.7.

213 Other requirements: Numpy (http://www.numpy.org), Scipy (http://scipy.org), Jellyfish

214 (http://www.cbcb.umd.edu/software/jellyfish), and Python programming language

215 (http://www.python.org).

216 License: GNU GPL3.

217 Any restrictions to use by non-academics: no special restrictions.

219 Acknowledgements:

We thank Dr. Peter Blomgren for help with the Advanced Numerical Analysis, and the

221 reviewers for their useful comments.

\section{References}

224

225

226

227

228

229

230

231

232

233

234

235

236

237

238

239
Altschul, S. F., T. L. Madden, A. A. Schäffer, J. Zhang, Z. Zhang, W. Miller, and D. J. Lipman, 1997: Gapped BLAST and PSI-BLAST: a new generation of protein database search programs. Nucleic Acids Res., 25, 3389-3402, doi:10.1093/nar/25.17.3389.

Aziz, R. K., and Coauthors, 2012: SEED Servers: High-Performance Access to the SEED Genomes, Annotations, and Metabolic Models. PLoS ONE, 7, e48053, doi:10.1371/journal.pone.0048053.

Belda-Ferre, P., L. D. Alcaraz, R. Cabrera-Rubio, H. Romero, A. Simón-Soro, M. Pignatelli, and A. Mira, 2012: The oral metagenome in health and disease. ISME J., 6, 46-56, doi:10.1038/ismej.2011.85.

Brady, A., and S. Salzberg, 2011: PhymmBL expanded: confidence scores, custom databases, parallelization and more. Nat. Methods, 8, 367-367, doi:10.1038/nmeth0511-367.

Carr, R., S. S. Shen-Orr, and E. Borenstein, 2013: Reconstructing the Genomic Content of Microbiome Taxa through Shotgun Metagenomic Deconvolution. PLoS Comput Biol, 9, e1003292, doi:10.1371/journal.pcbi.1003292.

Consortium, T. H. M. P., 2012: Structure, function and diversity of the healthy human microbiome. Nature, 486, 207-214, doi:10.1038/nature11234. 
Garda, B., and Z. Galias, 2012: Non-negative least squares and the Tikhonov regularization methods for coil design problems. 2012 International Conference on Signals and Electronic Systems (ICSES), 2012 International Conference on Signals and Electronic Systems (ICSES), 1-5.

Handelsman, J., 2004: Metagenomics: Application of Genomics to Uncultured Microorganisms. Microbiol. Mol. Biol. Rev., 68, 669-685, doi:10.1128/MMBR.68.4.669-685.2004.

Jones, E., T. Oliphant, and P. Peterson, 2001: SciPy: Open source scientific tools for Python. http://www.scipy.org/,. http://www.scipy.org/Citing_SciPy (Accessed October 23, 2013).

Kurokawa, K., and Coauthors, 2007: Comparative metagenomics revealed commonly enriched gene sets in human gut microbiomes. DNA Res. Int. J. Rapid Publ. Rep. Genes Genomes, 14, 169-181, doi:10.1093/dnares/dsm018.

Langmead, B., C. Trapnell, M. Pop, and S. L. Salzberg, 2009: Ultrafast and memory-efficient alignment of short DNA sequences to the human genome. Genome Biol., 10, R25, doi:10.1186/gb-2009-10-3-r25.

Lawson, C., and R. J. Hanson, 1987: Solving Least Squares Problems. SIAM,.

Lindner, M. S., and B. Y. Renard, 2013: Metagenomic abundance estimation and diagnostic testing on species level. Nucleic Acids Res., 41, e10, doi:10.1093/nar/gks803.

Mande, S. S., M. H. Mohammed, and T. S. Ghosh, 2012: Classification of metagenomic sequences: methods and challenges. Brief. Bioinform., 13, 669-681, doi:10.1093/bib/bbs054.

Marçais, G., and C. Kingsford, 2011: A fast, lock-free approach for efficient parallel counting of occurrences of k-mers. Bioinformatics, 27, 764-770, doi:10.1093/bioinformatics/btr011.

Mavromatis, K., and Coauthors, 2007: Use of simulated data sets to evaluate the fidelity of metagenomic processing methods. Nat. Methods, 4, 495-500, doi:10.1038/nmeth1043.

McElroy, K. E., F. Luciani, and T. Thomas, 2012: GemSIM: general, error-model based simulator of next-generation sequencing data. BMC Genomics, 13, 74, doi:10.1186/1471-2164-1374.

McHardy, A. C., H. G. Martín, A. Tsirigos, P. Hugenholtz, and I. Rigoutsos, 2007: Accurate phylogenetic classification of variable-length DNA fragments. Nat. Methods, 4, 63-72, doi:10.1038/nmeth976.

McNair, K., and R. Edwards, GenomePeek - A tool for prokaryotic genome and metagenome analysis.

Meinicke, P., K. P. Aßhauer, and T. Lingner, 2011: Mixture models for analysis of the taxonomic composition of metagenomes. Bioinformatics, btr266, doi:10.1093/bioinformatics/btr266.

Meyer, F., and Coauthors, 2008: The metagenomics RAST server - a public resource for the automatic phylogenetic and functional analysis of metagenomes. BMC Bioinformatics, 9 , 386, doi:10.1186/1471-2105-9-386. 
277

278

279

280

281

282

283

284

285

286

287

288

289

290

291

292

293

294

295

296

297

298

299

300

301

302
Nalbantoglu, O. U., S. F. Way, S. H. Hinrichs, and K. Sayood, 2011: RAIphy: Phylogenetic classification of metagenomics samples using iterative refinement of relative abundance index profiles. BMC Bioinformatics, 12, 41, doi:10.1186/1471-2105-12-41.

Segata, N., L. Waldron, A. Ballarini, V. Narasimhan, O. Jousson, and C. Huttenhower, 2012: Metagenomic microbial community profiling using unique clade-specific marker genes. Nat. Methods, 9, 811-814, doi:10.1038/nmeth.2066.

Sharon, I., and J. F. Banfield, 2013: Genomes from Metagenomics. Science, 342, 1057-1058, doi:10.1126/science.1247023.

Strous, M., B. Kraft, R. Bisdorf, and H. E. Tegetmeyer, 2012: The binning of metagenomic contigs for microbial physiology of mixed cultures. Front. Microbiol., 3, 410, doi:10.3389/fmicb.2012.00410.

$\mathrm{Su}, \mathrm{X} ., \mathrm{J} . \mathrm{Xu}$, and K. Ning, 2012: Parallel-META: efficient metagenomic data analysis based on high-performance computation. BMC Syst. Biol., 6, S16, doi:10.1186/1752-0509-6-S1S16.

Teeling, H., J. Waldmann, T. Lombardot, M. Bauer, and F. O. Glöckner, 2004: TETRA: a webservice and a stand-alone program for the analysis and comparison of tetranucleotide usage patterns in DNA sequences. BMC Bioinformatics, 5, 163, doi:10.1186/1471-21055-163.

Trindade-Silva, A. E., and Coauthors, 2012: Taxonomic and Functional Microbial Signatures of the Endemic Marine Sponge Arenosclera brasiliensis. PLoS ONE, 7, e39905, doi:10.1371/journal.pone.0039905.

Whitman, W. B., D. C. Coleman, and W. J. Wiebe, 1998: Prokaryotes: The unseen majority. Proc. Natl. Acad. Sci., 95, 6578-6583.

Zhang, Z., S. Schwartz, L. Wagner, and W. Miller, 2000: A greedy algorithm for aligning DNA sequences. J. Comput. Biol. J. Comput. Mol. Cell Biol., 7, 203-214, doi:10.1089/10665270050081478. 


\section{Figure 1}

Figure 1

Workflow of the FOCUS program.

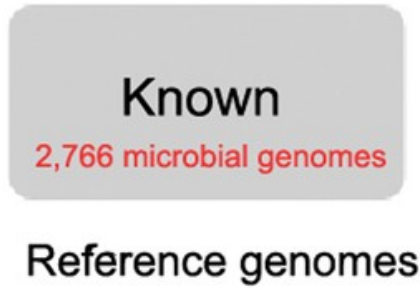

Precalculate k-mer

frequency for references

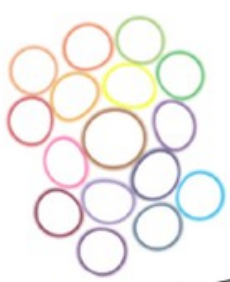

$\checkmark$

1) Calculate k-mer frequency for the input

\section{Unknown}

Metagenomes

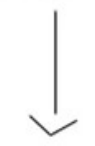

2) Find a non-negative vector $x \in \mathbb{R}^{n}$ to minimize the function

$$
f(x)=1 / 2\|A x-b\|^{2} \text {, where } x \geq 0 \text { and } \sum_{i=i}^{n} x_{i}=1
$$

List of "Known" genomes in the "Unknows" 


\section{Figure 2}

Figure 2

Genera-level taxonomy classification sorted by FOCUS prediction for the metagenome from a diseased human oral cavity using FOCUS, MetaPhIAn, MG-RAST, PhymnBL, RAlphy, Taxy, and FOCUS (mean). Error bars represent the standard deviation uncertainty in tested metagenome.

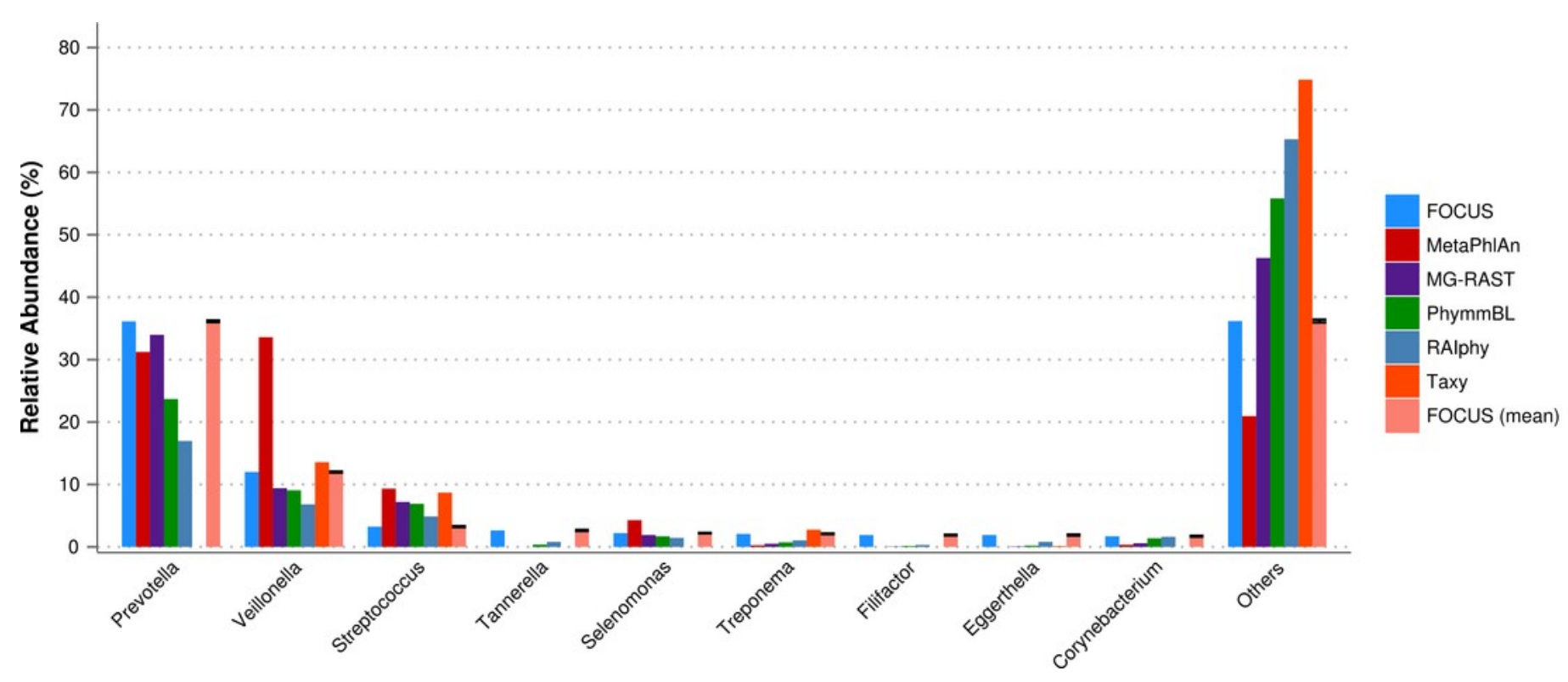




\section{Figure 3}

Figure 3

Scalability test using different sub-sets of the human oral cavity under disease metagenome using FOCUS, MetaPhIAn, MG-RAST, PhymnBL, RAlphy, Taxy.

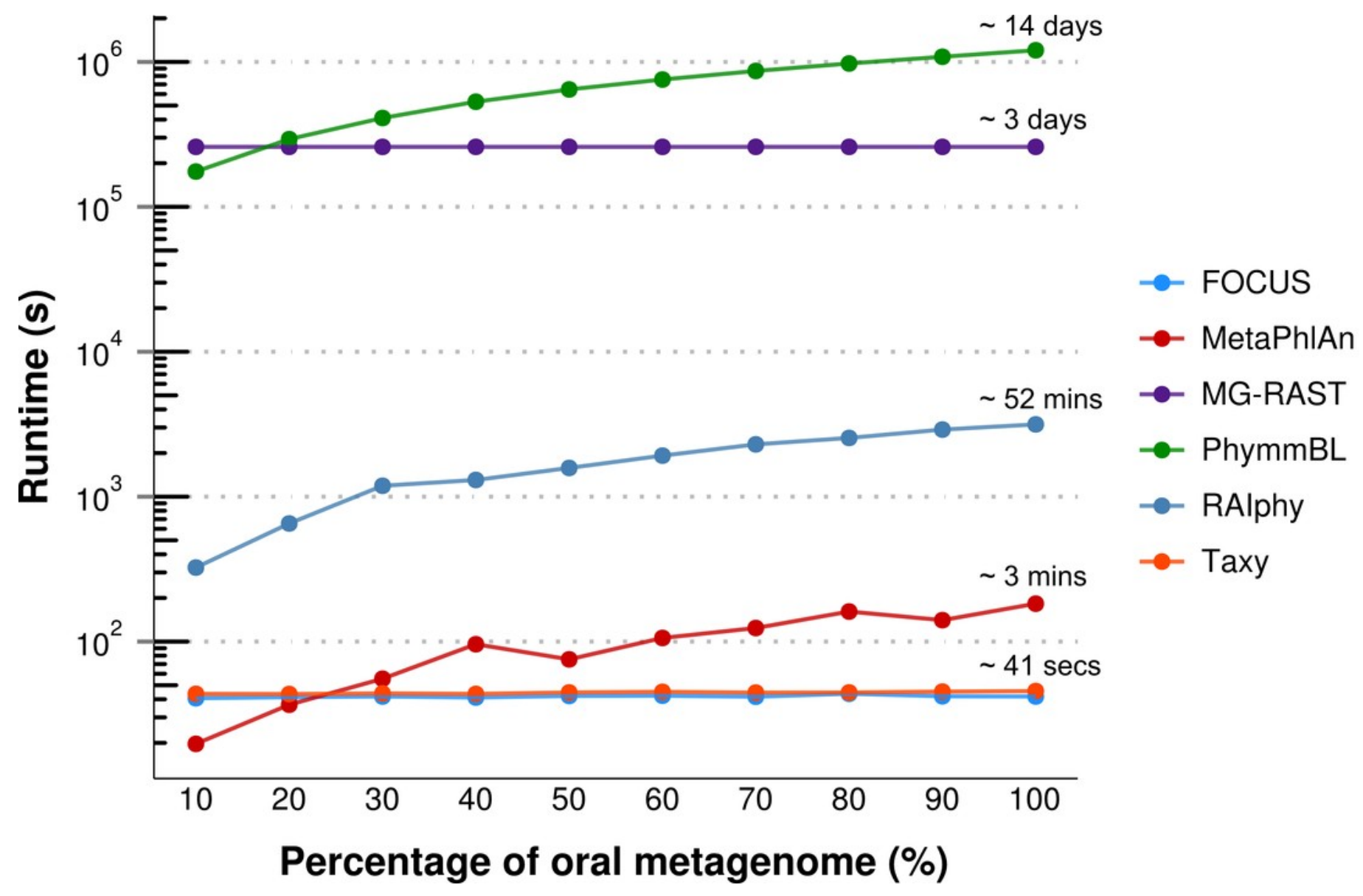




\section{Figure 4}

Figure 4

Genera-level taxonomy classification sorted by FOCUS prediction for the metagenome from a healthy human oral cavity using FOCUS, MetaPhIAn, MG-RAST, PhymnBL, RAlphy, Taxy, and FOCUS (mean). Error bars show the standard deviation uncertainty for the real metagenome.

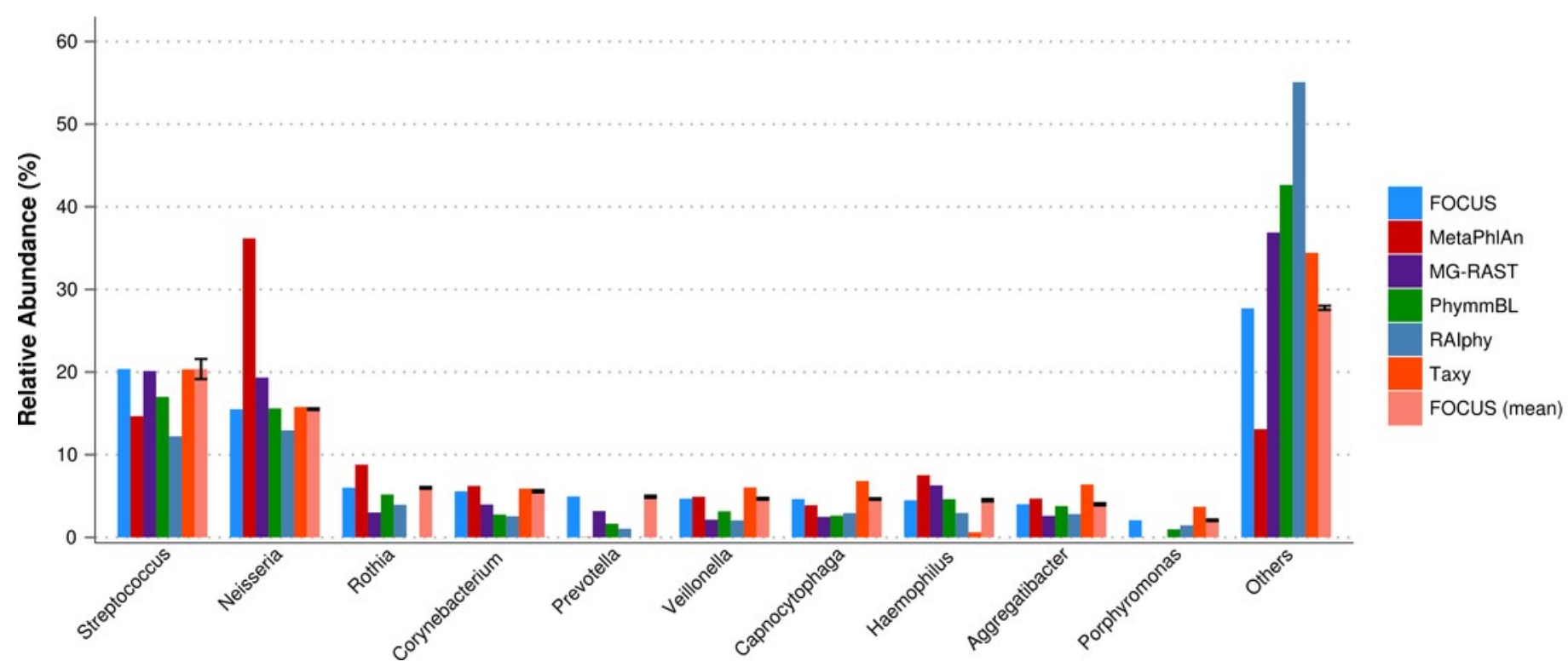




\section{Figure 5}

Figure 5

Genera-level taxonomy classification sorted by FOCUS prediction for the metagenome from a fecal metagenomic sample of a healthy human using FOCUS, MetaPhIAn, MG-RAST, PhymnBL, RAlphy, Taxy, and FOCUS (mean). Error bars show the standard deviation uncertainty for the real metagenome.

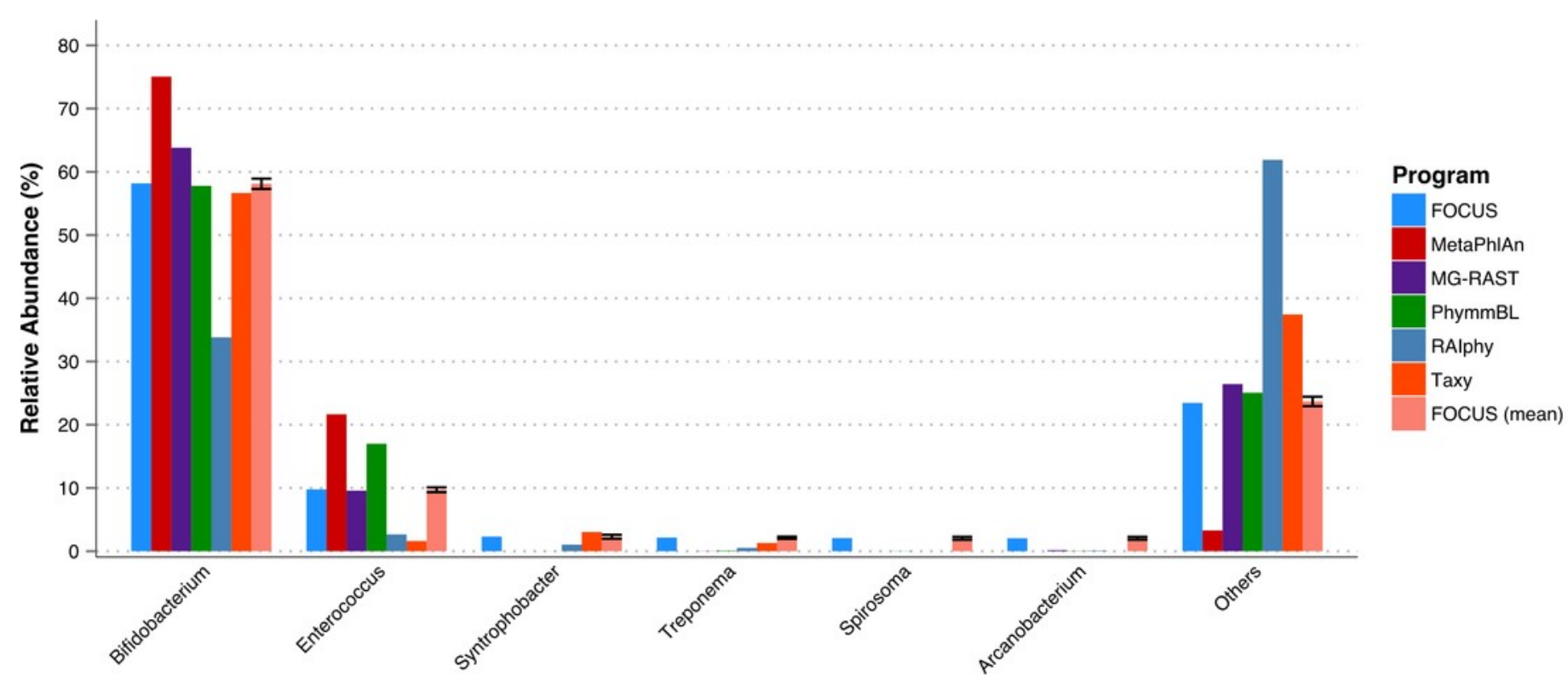




\section{Figure 6}

Figure 6

Heat-map representing the distance between the FOCUS and MetaPhIAn results for 300 metagenomes from the Human Microbiome Project across 15 body sites. The distance was computed using the Euclidean distance between the results of both tools.
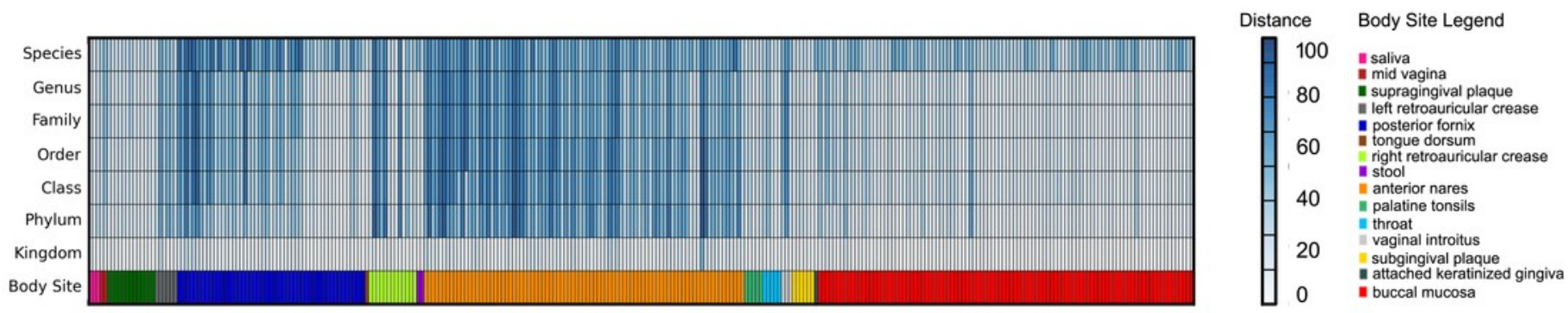


\section{Figure 7}

Figure 7

Genera-level taxonomy classification for the SimShort dataset using FOCUS, PhymnBL, RAlphy, and FOCUS (mean).

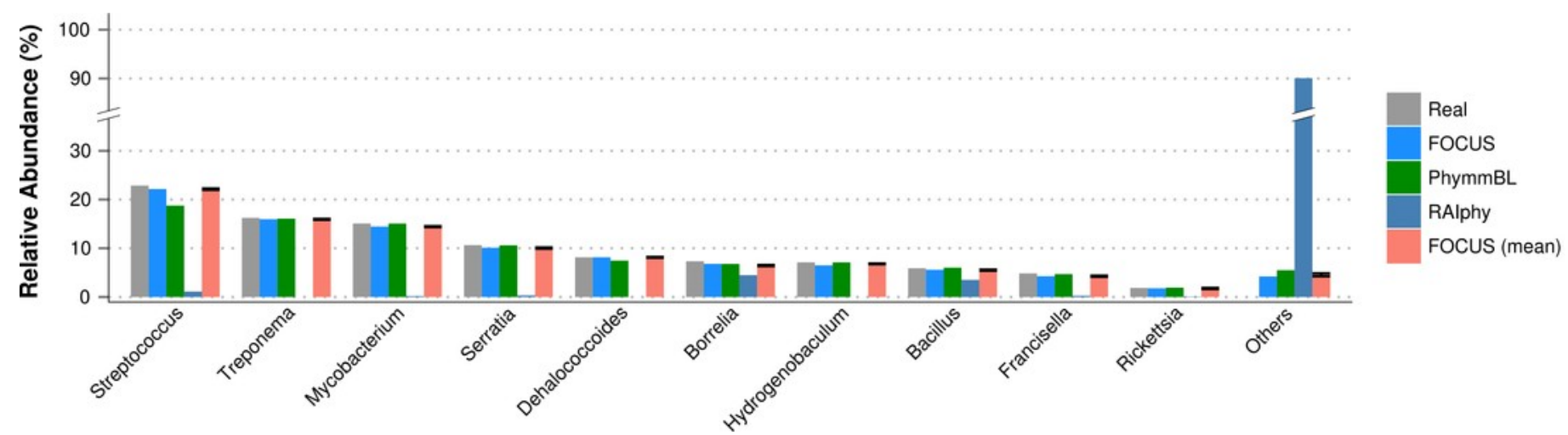




\section{Figure 8}

Figure 8

Class-level taxonomy classification for the SimHC dataset using FOCUS, PhymnBL, RAlphy, and FOCUS (mean).

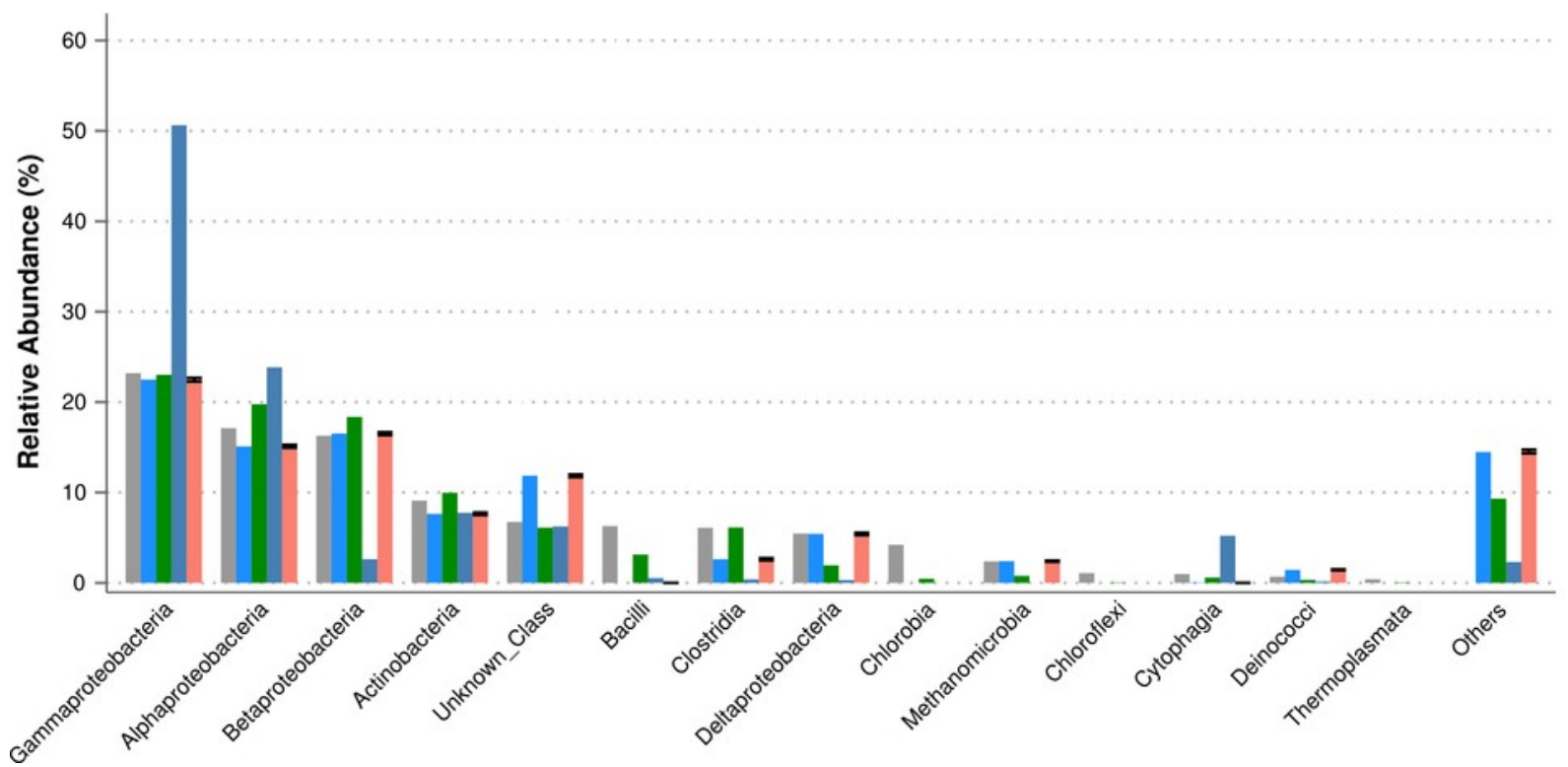




\section{Figure 9}

Figure 9

Genera-level taxonomy classification for the SimHC dataset using FOCUS, MetaPhIAn, MGRAST, PhymnBL, RAlphy, Taxy, GASiC, and FOCUS (mean).

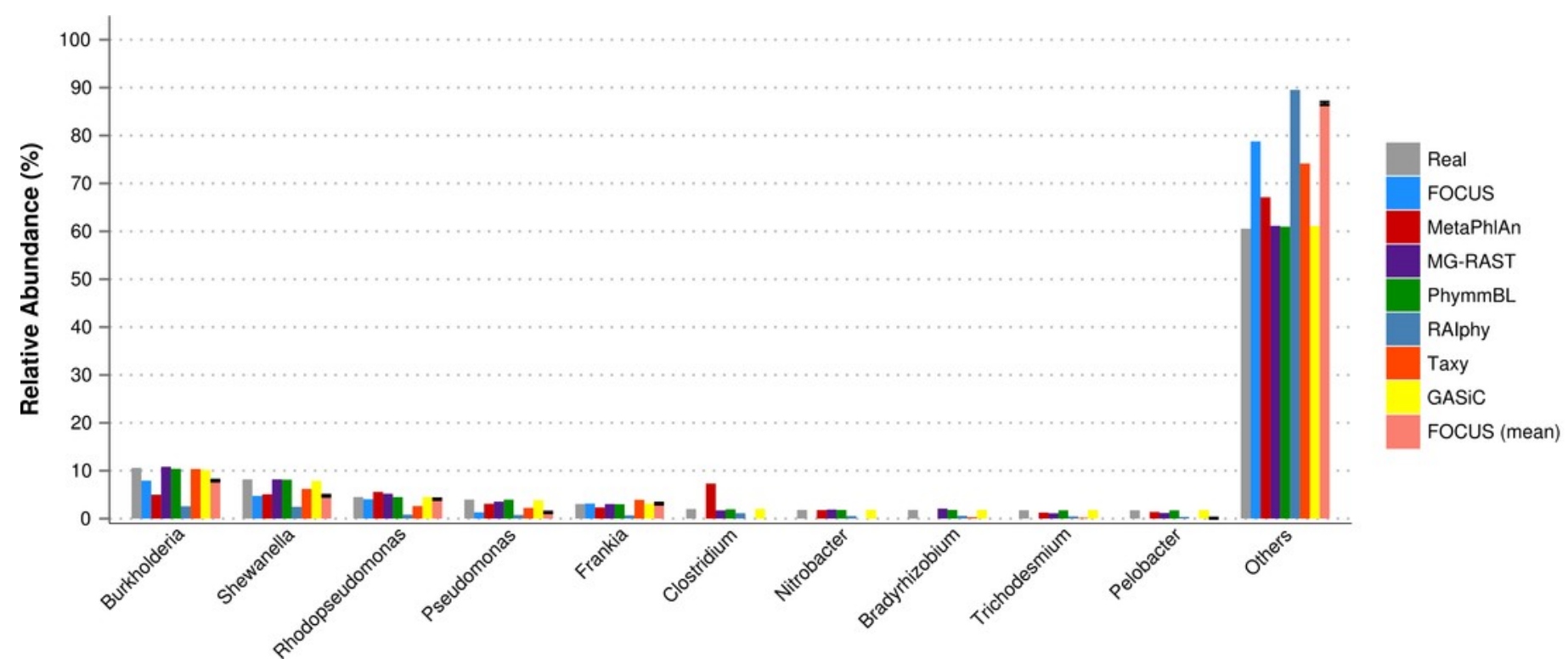




\section{Figure 10}

Figure 10

Numerical evaluation between the real and predicted abundance for the synthetic metagenomes computed by the Euclidean distance between the real and the predicted values.

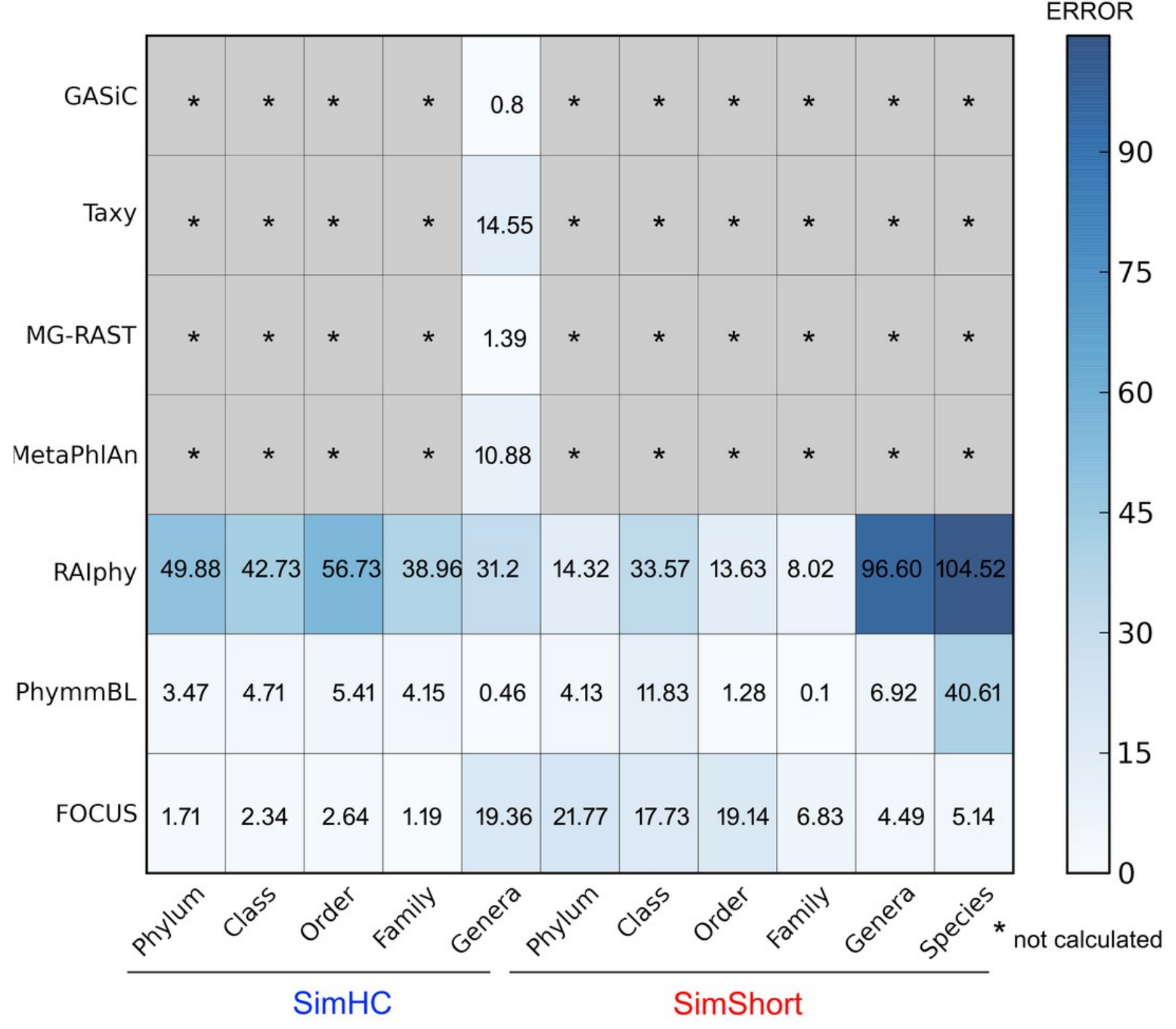

